\title{
Spherical Fuzzy Cost/Benefit Analysis of Wind Energy Investments
}

\author{
Sezi Cevik Onar ${ }^{(\bowtie)}$, Basar Oztaysi, and Cengiz Kahraman \\ Department of Industrial Engineering, Istanbul Technical University, \\ 34367 Istanbul, Turkey \\ \{cevikse,infus\}@itu.edu.tr
}

\begin{abstract}
Energy investments are highly risky due to the uncertainties of the energy market. Over the past two decades, the usage of wind energy has been increased dramatically. Appropriate wind energy investment valuations are vital for both the public and private sectors. The fluctuations in the energy prices, the uncertainties in the production levels and the changes in the investment costs harden the wind energy investment decision. Using the traditional investment valuations overlooks the necessary managerial flexibility of investments, uncertainty and risk concepts. The spherical fuzzy sets enable us dealing with this uncertain structure with the hesitancy. In this study, a new Spherical fuzzy cost benefit analysis is developed and modified to the wind energy investment evaluation. In order to show the applicability of the proposed method a wind energy investment is analyzed with it. This new approach by taking into account the hesitancy may enable investments to be evaluated with more flexibility and accuracy.
\end{abstract}

Keywords: Spherical fuzzy sets $\cdot$ Cost benefit analysis $\cdot$ Engineering economics $\cdot$ Wind energy investment analysis

\section{Introduction}

Wind energy is considered as one of the most important energy sources for the transition from fossil based traditional energy sources. In Europe, the total wind power investment is $€ 52$ billion in 2019 [1]. Yet, the amount of investments for the new projects is $€ 19$ billion which is less than in 2018. The improvements in the wind energy technologies along with the economies of scale, increased the capacity factor while decreasing the installation costs. In 2018, the capacity factor of wind energy investments raised to $35 \%$ from $26 \%$ in 2010 [2].

Although wind energy investments are reliable investments for the medium to long term, the crisis such as the crisis due to COVID-19 pandemic slow down the reliable energy investments. Modeling the uncertainty and hesitancy in wind energy investments become one of the biggest challenges for the decision makers. Figure 1 shows the change in the total installed costs, levelized cost of energy and the average capacity factors are given in Fig. 1 [2].

In literature, many studies focus on the modelling of uncertainty in energy investments. Dawis and Owens [3] utilized the real options analysis in estimating the 


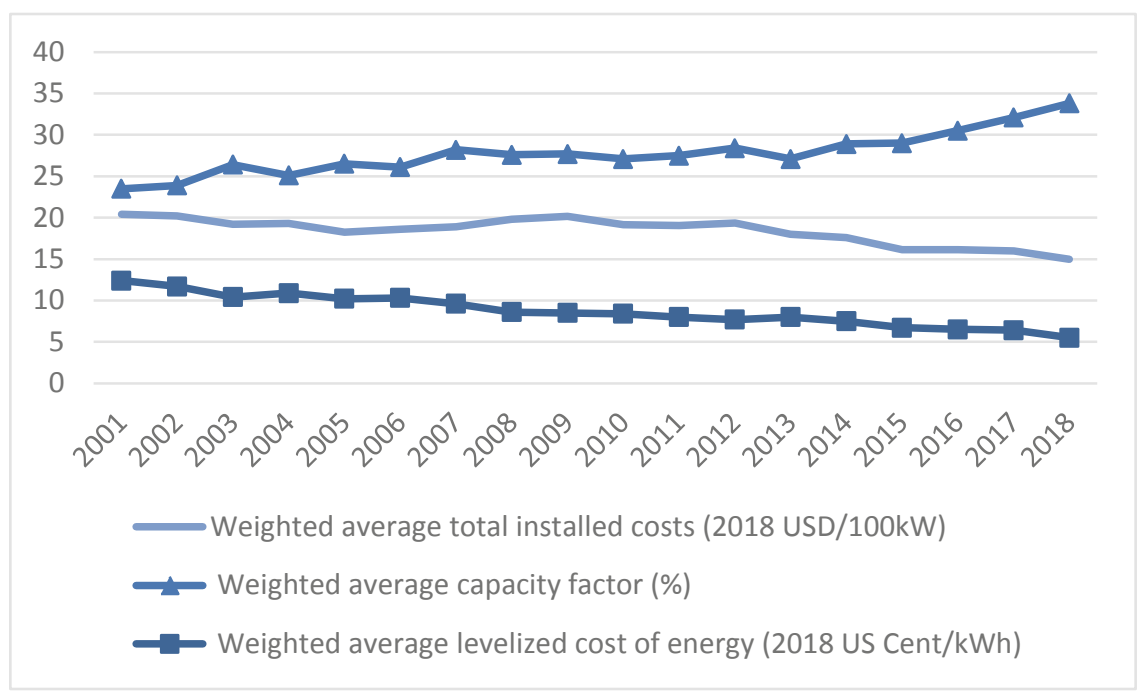

Fig. 1. The wind energy investments statistics [2].

value of renewable energy and electricity generating technologies exposed to variable fossil prices. The main findings of this study are that the real value of renewable energy investments cannot be obtained by using traditional valuation techniques, and highlevel valuation methods should be used. Similarly, Siddiqui et al. [4] utilized the real options analysis for the benefit of the renewable energy technology developments. The researchers emphasized that the deterministic analyzes ignore uncertainties in renewable energy costs, technical risks underlying R\&D processes, and the possibilities of regulation that should be made in proportion to the changing state of the world. Fuzzy sets have been widely used for modeling the uncertainty in renewable energy investments. Cevik Onar and Kilavuz [5] utilized real options for modelling wind energy investments in Turkey.

The fuzzy sets are also widely utilized for modelling the uncertainty and hesitancy in renewable energy investments [6-9]. Spherical fuzzy sets are excellent tools that can deal with hesitancy and uncertainty by enabling defining membership, nonmembership and hesitancy values independently. Cost benefit analysis is a useful engineering economics tool but it needs to be modified for dealing with uncertainty and hesitancy [10]. In this study, spherical fuzzy cost benefit analysis is utilized for evaluating wind energy investments.

The rest of the paper is organized as follows: The second section first gives the basic definitions of Spherical fuzzy sets. Section 3 gives the details of Spherical fuzzy Cost Benefit analysis. Section 4 applies the proposed methodology to a wind energy investment. Section 5 concludes and gives further suggestions. 


\section{Spherical Fuzzy Sets}

Spherical fuzzy developed by Gundogdu and Kahraman [11] can be defined as follows [11-14]:

Let $\widetilde{S p}$ be a Spherical fuzzy set on the universe U, it can be defined as in Eq. (2) and (3).

$$
\widetilde{S p}=\left\{\left\langle s,\left(\mu_{s p}(s), \vartheta_{s p}(s), \pi_{s p}(s)\right)\right\rangle \mid s \in U\right\}
$$

where $\mu_{s p}(s): U \rightarrow[0,1], \mu_{s p}(s): U \rightarrow[0,1], \mu_{s p}(s): U \rightarrow[0,1], \mu_{s p}(s)$ denotes the degree of membership, $\vartheta_{s p}(s)$ denotes the degree of membership and $\pi_{s p}(s)$ is the degree of non-membership and hesitancy of $s$ to $\widetilde{S p}$.

$$
0 \leq \mu_{s p}^{2}(s)+\vartheta_{s p}^{2}(s)+\pi_{s p}^{2}(s) \leq 1
$$

The basic operations on the Spherical fuzzy sets are given in Eq. (3-7) [3-7].

$$
\begin{aligned}
& \tilde{S} p_{1} \oplus \widetilde{S p}_{2}=\left(\mu_{s p 1}^{2}(s)+\mu_{s p 2}^{2}(s)-\mu_{s p 1}^{2}(s) \mu_{s p 2}^{2}(s)\right)^{0.5}, \vartheta_{s p 1}(s) \vartheta_{s p 2}(s) \\
& \left(\left(1-\mu_{s p 2}^{2}(s)\right) \pi_{s p 1}^{2}(s)+\left(1-\mu_{s p 1}^{2}(s)\right) \pi_{s p 2}^{2}(s)-\pi_{s p 1}^{2}(s) \pi_{s p 2}^{2}(s)\right)^{0.5} \\
& \tilde{S}_{p 1} \otimes \tilde{S}_{p 2}=\mu_{s p 1}(s) \mu_{s p 2}(s),\left(\vartheta_{s p 1}^{2}(s)+\vartheta_{s p 2}^{2}(s)-\vartheta_{s p 1}^{2}(s) \vartheta_{s p 2}^{2}(s)\right)^{0.5} \\
& \left(\left(1-\vartheta_{s p 2}^{2}(s)\right) \pi_{s p 1}^{2}(s)+\left(1-\vartheta_{s p 1}^{2}(s)\right) \pi_{s p 2}^{2}(s)-\pi_{s p 1}^{2}(s) \pi_{s p 2}^{2}(s)\right)^{0.5} \\
\lambda \odot \tilde{S}_{p}= & \left(\left(1-\left(1-\mu_{s p}^{2}(s)\right)^{\lambda}\right)\right)^{0.5}, \vartheta_{s p}(s)^{\lambda},\left(\left(1-\mu_{s p}^{2}(s)\right)^{\lambda}-\left(1-\mu_{s p}^{2}(s)-\pi_{s p}^{2}(s)\right)^{\lambda}\right)^{0.5}
\end{aligned}
$$

where $\lambda>0$.

Spherical fuzzy sets can be defuzzified by using the scoring function defined in Eq. (6).

$$
\text { Score } \widetilde{S p}=\left(\mu_{s_{p}}(s)-\pi_{s p}(s)\right)^{2}-\left(\vartheta_{s p}(s)-\pi_{s p}(s)\right)^{2}
$$

In order to aggregate and defuzzify the Spherical fuzzy sets Spherical Weighted Arithmetic Mean (SWAM) aggregation operator can be utilized. 


$$
\begin{aligned}
\operatorname{SWAM} & \left(\widetilde{S p}_{1}, \ldots, \widetilde{S p}_{n}\right)=w_{1} \tilde{S} p_{1}+\ldots+w_{n} \widetilde{S p}_{n}= \\
& \left\langle\left[\begin{array}{c}
\left(1-\prod_{i=1}^{n}\left(1-\mu_{s p i}^{2}(s)\right)^{w_{i}}\right)^{0.5}, \prod_{i=1}^{n} \vartheta_{s p i}(s)^{w_{i}}, \\
\left(\left(\prod_{i=1}^{n}\left(1-\mu_{s p i}^{2}(s)\right)^{w_{i}}\right)-\prod_{i=1}^{n}\left(1-\mu_{s p i}^{2}(s)-\pi_{s p i}^{2}(s)\right)^{w_{i}}\right)^{0.5}
\end{array}\right]\right\rangle
\end{aligned}
$$

where $w=\left(w_{1}, w_{2} \ldots . . ., w_{n}\right) ; w_{i} \in[0,1] ; \sum_{i=1}^{n} w_{i}=1$.

\section{Spherical Fuzzy Cost Benefit Analysis}

The engineering economic analysis can be enhanced by using fuzzy sets [10, 15]. In the Spherical fuzzy cost benefit analysis, Spherical numbers are utilized for defining the parameters. These parameters are initial funding $\left(\widetilde{I F}_{S p}\right)$, the uniform annual costs $\left(\widetilde{U A C}_{S p}\right)$, uniform annual income $\left(\widetilde{U A I}_{S p}\right)$, the life of the investment $\left(\widetilde{m}_{S p}\right)$, the interest rate $\left(\tilde{r}_{S p}\right)$, and the scrap value $\left(\widetilde{S V}_{S p}\right)$. In order to represent the hesitancy of the decision makers, spherical fuzzy values are assigned. These parameters can be defined as follows:

$$
\begin{aligned}
& \widetilde{I F}_{S p}=\left\{\left\langle i f_{1}, \widetilde{S p}_{1}\right\rangle,\left\langle i f_{2}, \widetilde{S p_{2}}\right\rangle, \ldots,\left\langle i f_{n}, \widetilde{S p_{n}}\right\rangle\right\} \\
& \widetilde{U A C}_{S p}=\left\{\left\langle u a c_{1}, \widetilde{S} p_{1}\right\rangle,\left\langle u a c_{2}, \widetilde{S p}_{2}\right\rangle, \ldots,\left\langle u a c_{n}, \widetilde{S p}_{n}\right\rangle\right\} \\
& \widetilde{U A I}_{S p}=\left\{\left\langle u a i_{1},{\widetilde{S p_{1}}}_{1},\left\langle u a i_{n}, \widetilde{S p}_{2}\right\rangle, \ldots,\left\langle u a i_{n}, \widetilde{S p}_{n}\right\rangle\right\}\right. \\
& \widetilde{S V}_{S p}=\left\{\left\langle s v_{1}, \widetilde{S p}_{1}\right\rangle,\left\langle s v_{2}, \widetilde{S p}_{2}\right\rangle, \ldots,\left\langle s v_{n}, \widetilde{S p}_{n}\right\rangle\right\} \\
& \widetilde{m}_{S p}=\left\{\left\langle m_{1}, \widetilde{S p}_{1}\right\rangle,\left\langle m_{2}, \widetilde{S p}_{2}\right\rangle, \ldots,\left\langle m_{n}, \widetilde{S p_{n}}\right\rangle\right\} \\
& \tilde{r}_{S p}=\left\{\left\langle r_{1}, \tilde{S} p_{1}\right\rangle,\left\langle r_{2}, \widetilde{S p_{2}}\right\rangle,\left\langle r_{n}, \tilde{S} p_{n}\right\rangle\right\}
\end{aligned}
$$

By using these parameters benefit/cost $\left(\frac{\tilde{B}_{S_{p}}}{\tilde{C}_{S_{p}}}\right)$ analysis of as single alternative can be defined as follows:

$$
\frac{\widetilde{B}_{S p}}{\tilde{C}_{S p}}=\frac{\widetilde{U A I}_{S p}\left(\frac{P}{A}, \tilde{r}_{S p}, \widetilde{m}_{S p}\right)}{\widetilde{I F}_{S p}+\widetilde{U A C}_{S p}\left(\frac{P}{A}, \tilde{r}_{S p}, \widetilde{m}_{S p}\right)-\widetilde{S V}_{S p}\left(\frac{P}{F}, \tilde{r}_{S p}, \widetilde{m}_{S p}\right)}
$$

or 


$$
\frac{\tilde{B}_{S p}}{\tilde{C}_{S p}}=\frac{\widetilde{U A I}\left[\begin{array}{c}
\left(1+\tilde{r}_{S p}\right)^{\tilde{m}_{S p}}-1 \\
\widetilde{I F}_{S p}+\widetilde{U A C}_{S p}\left[\frac{\left(1+\tilde{r}_{S p}\right)^{m_{S p}}-1}{\tilde{r}_{S p}\left(1+\tilde{r}_{S p}\right)^{m_{S p}}}\right]-\widetilde{S V}
\end{array}\right]}{\widetilde{r}_{S p}\left(1+\tilde{r}_{S p}\right)^{-\widetilde{m}_{S p}}}
$$

For an incremental Spherical cost benefit analysis can be calculated as follows:

$$
\frac{\widetilde{\Delta B_{S p}}}{\widetilde{\Delta C_{S p}}}=\frac{\widetilde{\Delta U A} I_{S p}\left(\frac{P}{A}, \tilde{r}_{S p}, \widetilde{m}_{S p}\right)}{\widetilde{\Delta I F}_{S p}+\widetilde{\Delta U A} C_{S p}\left(\frac{P}{A}, \tilde{r}_{S p}, \widetilde{m}_{S p}\right)-\widetilde{\Delta S V_{S p}\left(\frac{P}{F}, \tilde{r}_{S p}, \widetilde{m}_{S p}\right)}}
$$

or

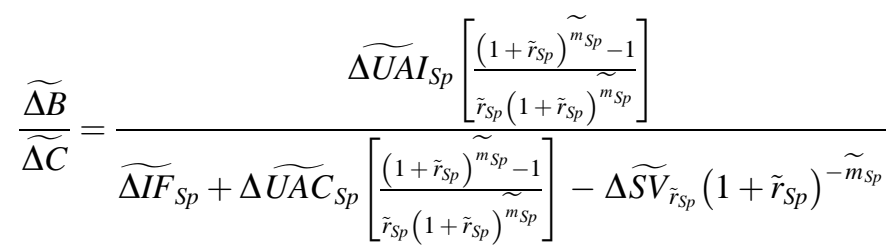

Where

$$
\begin{aligned}
& \widetilde{I F}_{S p}=\bigcup_{j=1}^{n} \boldsymbol{S W A M}\left\{\left\langle i f_{1}, \widetilde{S p}_{1}\right\rangle,\left\langle i f_{2}, \widetilde{S p_{2}}\right\rangle, \ldots,\left\langle i f_{n}, \widetilde{S p}_{n}\right\rangle\right\} \\
& \widetilde{U A C}_{S p}=\bigcup_{j=1}^{n} \boldsymbol{S W A M}\left\{\left\langle u a c_{1}, \widetilde{S p_{1}}\right\rangle,\left\langle u a c_{2}, \tilde{S} p_{2}\right\rangle, \ldots,\left\langle u a c_{n}, \widetilde{S}_{n}\right\rangle\right\} \\
& \widetilde{U A I}_{S p}=\bigcup_{j=1}^{n} \boldsymbol{S W A M}\left\{\left\langle u a i_{1}, \tilde{S} p_{1}\right\rangle,\left\langle u a i_{2}, \widetilde{S p_{2}}\right\rangle, \ldots,\left\langle u a i_{n}, \tilde{S}_{n}\right\rangle\right\} \\
& \widetilde{S V}_{S}=\bigcup_{j=1}^{n} \boldsymbol{S W A M}\left\{\left\langle s v_{1}, \tilde{S}_{p_{1}}\right\rangle,\left\langle s v_{2}, \widetilde{S p_{2}}\right\rangle, \ldots,\left\langle s v_{n}, \widetilde{S p_{n}}\right\rangle\right\} \\
& \widetilde{m}_{S p}=\bigcup_{j=1}^{n} \boldsymbol{S W A M}\left\{\left\langle m_{1}, \widetilde{S p_{1}}\right\rangle,\left\langle m_{2}, \widetilde{S p_{2}}\right\rangle, \ldots,\left\langle m_{n}, \widetilde{S p_{n}}\right\rangle\right\} \\
& \tilde{r}_{S}=\bigcup_{j=1}^{n} \boldsymbol{S W A M}\left\{\left\langle r_{1}, \tilde{S}_{1}\right\rangle,\left\langle r_{2}, \widetilde{S p_{2}}\right\rangle, \ldots,\left\langle r_{n}, \tilde{S}_{n}\right\rangle\right\}
\end{aligned}
$$

\section{Application}

A wind energy investment in the Marmara Region of Turkey are evaluated by using the proposed methodology. Three experts evaluate a wind tribune alternative, the weights of the decision makers are $0.4,0.4$ and 0.2 . The possible values of the alternatives and the evaluations done by the experts are given in Table 1 .

The aggregated values are defuzzified and summed by the proposed methodology. Table 2 gives the defuzzified investment parameters.

The benefit $\tilde{B}_{S p}=\$ 10,052,820$ and the cost $\tilde{C}_{S p}=\$ 6,291,507.84$ of the wind energy investment are calculated by using the proposed approach. By using the these parameters benefit/cost $\frac{\tilde{B}_{S p}}{\tilde{C}_{S p}}=1.598$ is calculated. This wind energy investment is a favorable investment. 
Table 1. Spherical fuzzy wind investment parameters.

\begin{tabular}{|c|c|c|c|c|}
\hline \multirow[t]{3}{*}{ Parameters } & \multirow[t]{3}{*}{ Values } & \multicolumn{3}{|c|}{ Experts' evaluations } \\
\hline & & E1 & E2 & E3 \\
\hline & & 0.4 & 0.4 & 0.2 \\
\hline \multirow[t]{3}{*}{$\widetilde{I F}_{S p}$} & $\$ 2,000,000$ & $(0.8,0.2,0.2)$ & $(0.7,0.3,0.3)$ & $(0.6,0.4,0.4)$ \\
\hline & $\$ 2,500,000$ & $(0.6,0.4,0.4)$ & $(0.8,0.2,0.2)$ & $(0.7,0.3,0.3)$ \\
\hline & $\$ 3,000,000$ & $(0.4,0.6,0.4)$ & $(0.5,0.5,0.5)$ & $(0.6,0.4,0.4)$ \\
\hline \multirow[t]{3}{*}{$\widetilde{U A C}_{S p}$} & $\$ 330,000$ & $(0.5,0.5,0.5)$ & $(0.6,0.4,0.4)$ & $(0.7,0.3,0.3)$ \\
\hline & $\$ 340,000$ & $(0.4,0.6,0.4)$ & $(0.8,0.2,0.2)$ & $(0.4,0.6,0.4)$ \\
\hline & $\$ 350,000$ & $(0.7,0.3,0.3)$ & $(0.7,0.3,0.3)$ & $(0.9,0.1,0.1)$ \\
\hline \multirow{3}{*}{$\widetilde{U A I}_{S p}$} & $\$ 850,000$ & $(0.4,0.6,0.4)$ & $(0.2,0.8,0.2)$ & $(0.8,0.2,0.2)$ \\
\hline & $\$ 900,000$ & $(0.6,0.4,0.4)$ & $(0.5,0.5,0.5)$ & $(0.4,0.6,0.4)$ \\
\hline & $\$ 950,000$ & $(0.3,0.7,0.3)$ & $(0.7,0.3,0.3)$ & $(0.6,0.4,0.4)$ \\
\hline \multirow[t]{3}{*}{$\widetilde{S V}_{S}$} & $\$ 4,000$ & $(0.5,0.5,0.5)$ & $(0.7,0.3,0.3)$ & $(0.5,0.5,0.5)$ \\
\hline & $\$ 5,000$ & $(0.4,0.6,0.4)$ & $(0.8,0.2,0.2)$ & $(0.9,0.1,0.1)$ \\
\hline & $\$ 6,000$ & $(0.7,0.3,0.3)$ & $(0.5,0.5,0.5)$ & $(0.7,0.3,0.3)$ \\
\hline \multirow[t]{3}{*}{$\tilde{r}_{S}$} & $5 \%$ & $(0.4,0.6,0.4)$ & $(0.4,0.6,0.4)$ & $(0.3,0.7,0.3)$ \\
\hline & $6 \%$ & $(0.2,0.8,0.2)$ & $(0.4,0.6,0.4)$ & $(0.5,0.5,0.5)$ \\
\hline & $7 \%$ & $(0.7,0.3,0.3)$ & $(0.7,0.3,0.3)$ & $(0.4,0.6,0.4)$ \\
\hline \multirow[t]{3}{*}{$\widetilde{m}_{S p}$} & 18 & $(0.5,0.5,0.5)$ & $(0.5,0.5,0.5)$ & $(0.9,0.1,0.1)$ \\
\hline & 19 & $(0.8,0.2,0.2)$ & $(0.7,0.3,0.3)$ & $(0.6,0.4,0.4)$ \\
\hline & 20 & $(0.2,0.8,0.2)$ & $(0.3,0.7,0.3)$ & $(0.5,0.5,0.5)$ \\
\hline
\end{tabular}

Table 2. Defuzzified values of the investment parameters.

\begin{tabular}{l|l}
\hline Parameters & Defuzzified values \\
\hline$\widetilde{I F}_{S p}$ & $\$ 2,508,536$ \\
\hline$\widetilde{U A C}_{S p}$ & $\$ 340,080$ \\
$\widetilde{U A I}_{S p}$ & $\$ 900,154$ \\
$\widetilde{S V}_{S}$ & $\$ 4,986$ \\
\hline$\widetilde{r}_{S}$ & $5.98 \%$ \\
\hline$\widetilde{m}_{S p}$ & 18.98 \\
\hline
\end{tabular}

\section{Conclusion and Further Suggestions}

Wind energy investments are one of the fastest-increasing investment areas in renewable energy. The installed wind capacity has been increasing not only due to the increasing awareness on the sustainability and the consequent governmental supports but also due to the decrease in the costs. Wind energy production is available and has great potential in many parts of the world. Despite wind energy has huge potential, wind energy investments are hard to evaluate. Fuzzy sets are excellent tools for dealing 
with uncertainty $[16,17]$. Spherical fuzzy sets, one of the newest extensions of fuzzy sets enable us to deal with uncertainty and hesitancy. In this study, we propose a novel spherical fuzzy cost/benefit analysis for evaluating uncertain wind energy investments. The applicability of the model is shown by applying it to a wind energy investment decision.

For further studies, several wind energy investments can be compared by using the proposed cost/benefit analysis. The results of the study can be compared with the other engineering economic analysis. A sensitivity analysis can be applied to see the effect of decision maker weights in the evaluation results.

\section{References}

1. Wind Europe Report. https://windeurope.org/about-wind/reports/financing-and-investmenttrends-2019/\#findings. Accessed 20 Apr 2020

2. Irene Stats. https://www.irena.org/publications/2019/May/Renewable-power-generationcosts-in-2018\#RestrictedModal. Accessed 20 Apr 2020

3. Davis, G., Owens, B.: Optimizing the level of renewable electric R\&D expenditures using real options analysis. Energy Policy 31(15), 1589-1608 (2003)

4. Siddiqui, A.S., Marnay, C., Wiser, R.H.: Real options valuation of US federal renewable energy research, development, demonstration, and deployment. Energy Policy 35(1), 265279 (2007)

5. Cevik Onar, S., Kilavuz, T.N.: Risk analysis of wind energy investments in Turkey. Hum. Ecol. Risk Assess. 21(5), 1230-1245 (2015)

6. Çoban, V., Onar, S.Ç.: Modelling solar energy usage with fuzzy cognitive maps. Intell. Syst. Ref. Libr. 113, 159-187 (2017)

7. Çoban, V., Cevik Onar, S.: Energy management maturity model based on fuzzy logic. Adv. Intell. Syst. Comput. 1029, 1034-1041 (2020)

8. Onar, S.C., Oztaysi, B., Otay, I., Kahraman, C.: Multi-expert wind energy technology selection using interval-valued intuitionistic fuzzy sets. Energy 90, 274-285 (2015)

9. Çoban, V., Çevik Onar, S.: Pythagorean fuzzy engineering economic analysis of solar power plants. Soft. Comput. 22, 5007-5020 (2018)

10. Kahraman, C., Onar, S.C., Oztaysi, B.: A comparison of wind energy investment alternatives using interval-valued intuitionistic fuzzy benefit/cost analysis. Sustainability 8(2), 118 (2016)

11. Kutlu Gündoğdu, F., Kahraman, C.: Spherical fuzzy sets and spherical fuzzy TOPSIS method. J. Intell. Fuzzy Syst. 36(1), 337-352 (2019)

12. Gündoğdu, F.K.: Principals of spherical fuzzy sets. Adv. Intell. Syst. Comput. 1029, 15-23 (2020)

13. Gündoğdu, F.K., Kahraman, C.: Extension of codas with spherical fuzzy sets. J. MultipleValued Logic Soft Comput. 33(4-5), 481-505 (2019)

14. Gündoğdu, F.K., Kahraman, C.: A novel spherical fuzzy analytic hierarchy process and its renewable energy application. Soft. Comput. 24, 4607-4621 (2020)

15. Kahraman, C., Çevik Onar, S., Öztayși, B.: Engineering economic analyses using intuitionistic and hesitant fuzzy sets. J. Intell. Fuzzy Syst. 29(3), 1151-1168 (2015) 
16. Estrella, F.J., Onar, S.C., Rodríguez, R.M., Oztaysi, B., Martínez, L., Kahraman, C.: Selecting firms in University technoparks: a hesitant linguistic fuzzy TOPSIS model for heterogeneous contexts. J. Intell. Fuzzy Syst. 33(2), 1155-1172 (2017)

17. Cevik Onar, S., Oztaysi, B., Kahraman, C.: Multicriteria evaluation of cloud service providers using Pythagorean fuzzy TOPSIS. J. Multiple-Valued Logic Soft Comput. 30(23), 263-283 (2018) 